\title{
La nature mise en spectacle
}

Aspects esthétiques et éthiques de la restauration des écosystèmes

Jean-Louis Fabiani

\section{CpenEdition}

\section{Journals}

Édition électronique

URL : https://journals.openedition.org/pa/1678

DOI : $10.4000 /$ pa. 1678

ISSN : 2273-0362

Éditeur

Université Lumière Lyon 2

Édition imprimée

Date de publication : 1 janvier 2003

Pagination : 23-29

ISBN : $1643-7706$

ISSN : $1634-7706$

Référence électronique

Jean-Louis Fabiani, «La nature mise en spectacle », Parcours anthropologiques [En ligne], 3 | 2003, mis en ligne le 06 juillet 2021, consulté le 21 juillet 2021. URL : http://journals.openedition.org/pa/1678 DOI : https://doi.org/10.4000/pa.1678 


\section{LA NATURE MISE EN SPECTACLE ASPECTS ESTHÉTIQUES ET ÉTHIQUES DE LA RESTAURATION DES ÉCOSYSTEMMES}

Les entreprises de restauration, de réhabilitation, voire de recréation d'écosystèmes ont connu un développement notable aux cours de ces dernières années: bien que la définition et l'ampleur des projets varient considérablement, et que le développement de ces pratiques soit très contrasté en fonction des conjonctures nationales (qu'on considere leur dimension scientifique ou institutionnelle), la dynamique en cours est particulièrement frappante, plus encore peut-être par les débats qu'elle suscite que par les opérations de terrain qu'elle engage. L'écologie de la restauration apparaît comme une sousdiscipline en croissance rapide dans le champ de la biologie de la conservation : il s'agit de réhabiliter ou de rétablir des espèces, des populations et des systèmes dégradés ou condamnés par les usages sociaux multiples des ressources naturelles accumulées à travers l'histoire. Les objets que traite cette sous-discipline se situent à l'intersection de préoccupations économiques et sociales et de préoccupations biologiques. Préoccupations sociales d'abord: les objectifs écosystémiques ne peuvent être dissociés en effet de l'emprise spatiale différentielle des activités économiques, de la constitution progressive d'une politique de gestion des ressources naturelles, des formes contrastées que prend la demande sociale d'espaces récréatifs et des transformations qui affectent les définitions sociales de la nature. Préoccupations biologiques ensuite : celles-ci présentent à leur tour un caractère croisé : nous sommes à l'évidence dans le cadre d'une missionoriented science (BOWLES and WHELAN 1996, p. 1), qui implique le développement d'une ingénierie appropriée et la constitution de savoirfaire appliqués. Simultanément, le type d'intervention sur le terrain qu'exige l'écologie de la restauration fournit une opportunité pour éprouver la validité des théories écologiques fondamentales (JORDAN et al. 1987). Le territoire d'une éco-technologie appliquée constitue un espace de test inédit pour les théories, espace dont certains pensent qu'il doit être privilégié du fait des expérimentations "en vraie grandeur " qu'il permet.

L'impératif de conservation n'est jamais la traduction pure et simple de normes biologiques : il s'agit inévitablement du produit d'une négociation sur la place à accorder aux valeurs " naturelles " dans les sociétés contemporaines. Le débat porte sur les moyens de déterminer la valeur économique des biens naturels ou sur la capacité de les soustraire a toute forme de calcul économique au nom de principes supérieurs. Comme le fait remarquer Joël Brown, l'écologie de la restauration est presque toujours confrontée à des stratégies concurrentes d'occupation de l'espace : dans la pratique, c'est lorsque les usages alternatifs n'of'frent pas d'attrait social ou économique particulier que la restauration est préférée (BOWLES and WHELAN 1996 p. 357).

L'écologie de la restauration apparaît donc comme un domaine largement ouvert aux controverses. Il existe un premier type de controverse, interne a la discipline : quand le biologiste doit-il intervenir, et avec quelle puissance d'intervention? Quelle doit être la durée de son action? Quels sont les critères d'une intervention réussie? Quels sont les indicateurs biologiques qui permettent de déterminer avec précision l'objectif à atteindre ? Quels sont, si l'on considère le caractère récent du développement de ces techniques, les critères d'évaluation qui permettent d'affirmer que l'objectif a été durablement atteint ? On connaît surtout les controverses qui ont lieu a propos de la réintroduction d'espèces animales, mais il en existe bien d'autres, moins visibles mais qui portent le plus souvent sur la détermination du caractère indigène (" native ") ou historique d'un élément du système ou de l'ensemble du système. Ces controverses savantes peuvent être reprises et relayées dans des cercles plus larges : les prises de position contradictoires des biologistes peuvent être mobilisées comme des ressources pour un deuxième type de controverse, qui se développe dans le monde social, à propos de la légitimité.de ces interventions restauratrices. A ce titre la branche de la biologie de la conservation sur laquelle nous portons notre attention constitue une excellente illustration du haut degré d'incertitude qui caractérise le contexte de la décision en matière de protection. Les thèmes esthétiques et éthiques sont inextricablement liés aux démarches des biologistes et à l'espace de la décision politique dans lequel ils se trouvent pris. On peut examiner successivement ces deux dimensions.

\section{Art, nature, plaisir esthétique}

La biologie de la conservation se préoccupe de maintenir la bio-diversité, et non pas la belle diversité. Il peut être justifié de se poser la question de savoir si un système restauré a nécessairement plus de valeur esthétique qu'un paysage dégradé par des siècles de mésusage. La généralisation d'une certaine forme de relativisme culturel qui conduit au constat de la multiplicité irréductible de codes esthétiques liés à la diversité des expériences du monde social et des conditionnements culturels, rend cette question moins étrange qu'on ne pourrait le penser 
d'emblée. On sait en outre que le statut esthétique de divers paysages naturels a fortement changé au cours des temps. Exemples les plus connus, le littoral et la montagne qui sont passés de la stigmatisation à la valorisation, de l'horrible au grandiose. Mais on pourrait évoquer les très nombreuses réticences d'ordre culturel au spectacle qu'offrent les zones humides, pourtant écologiquement valorisées). On admet cependant que dans la longue durée la valeur esthétique affectant les paysages s'est principalement constituée autour de la disparition du travail humain dans le champ de vision du spectateur. "Une terre qu'on travaille n'est presque jamais un paysage ", disait Raymond Williams (WILLIAMS 1973). La dégradation et la fragmentation qu'induisent les usages industriels de l'espace conduisent inévitablement à une déqualification des lieux (FABIANI 1996b) : l'exaltation de la beauté qu'engendre une forte emprise industrielle sur la nature constitue une exception (l'exemple des lignes à haute tension vient immédiatement à l'esprit pour illustrer l'unanimité d'une répulsion). Lorsque se développe cette position exceptionnelle, c'est le plus souvent sous la forme de la provocation artistique (ainsi les futuristes italiens qui préféraient la banlieue industrielle de Mestre à Venise) ou du défi philosophique (à travers la défense de l'industrialisme, ou la critique de l'antiindustrialisme). On pourrait dire que l'esthétisation de la nature exige à son principe quelque chose comme une désanthropisation, effective ou fictionnelle. Le cas du parc à l'anglaise est un bon exemple de l'invention d'une nature ludique faussement réensauvagée et dans laquelle le travail humain aussi bien que le travail spécifique du créateur de jardin ne doivent pas se voir, même s'il sont omniprésents. Dans les pratiques de consommation touristique, la surfréquentation des sites conduit toujours à leur dévalorisation à terme, indépendamment de l'évaluation de ses conséquences écologiques.

Il semble aller de soi qu'un écosystème non dégradé apporte en tant que tel une plus-value paysagère. La beauté est ici classiquement liée à la fonctionnalité d'un ensemble (est beau ce qui marche et qui répond adéquatement à une fonction). Mais il est clair que pour la plupart des spectateurs profanes, il ne s'agit pas d'observer des réseaux trophiques ou des systèmes populationenvironnement : le fonctionnement écosystémique ne se donne pas à voir, sauf par des médiations savantes qui restent encore tout à fait minoritaires. En outre, dans la mesure où les paysages esthétisés par des traditions culturelles sont en règle générale des constructions sociales complexes, dans lesquelles la nature peut être diversement (et parfois violemment) forcée, on ne peut pas conclure de manière simple de l'existence d'un écosystème qu'il a une valeur esthétique, tant celle-ci est liée à des codes qui ne valorisent pas pour elle-même la productivité écologique. Ajoutons que de nombreuses espèces (végétales et surtout animales) font l'objet de préventions ou de répulsions diverses pour convaincre que ce ne sont pas des ensembles naturels qui font l'objet de l'investissement esthétique, mais des productions sociales dans lesquelles l'ordre qui est donné à voir n'est presque jamais écologique.

Un écosystème n'est pas nécessairement beau à voir, précisément parce qu'il n'est pas d'emblée donné à voir. Il convient ici de distinguer entre le paysage tel que l'aborde majoritairement l'écologie du paysage, c'est-à-dire à travers une morphologie de l'environnement appuyée sur le recueil de données objectives concernant la géographie ou l'écologie, comme le remarque Augustin Berque dans un ouvrage récent (BERQUE 1995, p. 22) et le paysage qui est saisi par la médiation d'un processus de construction sociale du sens. Le partage ne se fait pas, comme on le croit souvent entre un ensemble de données objectives et une appropriation subjective (qu'elle soit le produit des déterminations biologiques de l'appareil sensoriel ou d'une histoire individuelle importe peu sous ce rapport), mais entre la description d'un système de relations portant sur des éléments naturels et une construction symbolique. La notion de prise paysagère proposée par Augustin Berque est très utile pour rendre compte du processus interprétatif qui permet de reconnaître et de qualifier des paysages : les prises paysagères sont des " motifs doués de stabilité - d'une relative stabilité - lesquels tendent à se reproduire, à se représenter dans chaque nouveau paysage " (p. 32). Les "écosymboles" sont des entités ambivalentes qui articulent la dimension écologique et la dimension symbolique. Mais pour qu'un écosymbole accède à la "condition paysagère ", il est nécessaire, remarque encore Augustin Berque, "qu'un certain ensemble de représentations les configure en un schème esthétique explicitement paysager " (p. 34). En d'autres termes, tout écosystème, ou tout écocomplexe (entendu comme agencement d'écosystèmes) ne constitue pas automatiquement un paysage.

Les diverses formes d'investissement esthétique ou moral dont la nature est l'objet sont le plus souvent liées à la valorisation d'espèces ou d'espaces. On pense ainsi au privilège extraordinaire qu'ont les oiseaux (qu'il s'agisse d'observation ou de protection): il existe des espèces qui sont des espèces-clés au sens social du terme. Ce ne sont pas ici des interactions, et encore moins un système, qui font l'objet de l'attention, mais des espèces qui constituent le support d'investissements symboliques variés. Il faudrait sans doute se donner les moyens d'une vérification empirique, mais il semble que l'observation des animaux, même si elle est scientifiquement armée, privilégie des trajectoires individuelles ou des considérations esthétiques par rapport aux interactions. Il y a dans cette valorisation sélective des espèces et des espaces l'imposition d'une grille de lecture de la nature dont on peut supposer qu'elle est faiblement connectée à la connaissance effective des interactions dans l'écosystème, ou même à un désir de connaissance de ces interactions.

La question de la valeur esthétique d'un écosystème peut être abordée d'un autre point de vue. $\mathrm{Si}$ ce que nous valorisons dans la contemplation de la nature, c'est avant tout le fait qu'elle offre à notre regard des compositions d'éléments non modifiés par l'activité humaine, pouvons-nous apprécier de la même façon des ensembles naturels "authentiques" et des ensembles dont l'authenticité a été rétablie ou reconstruite par l'intervention d'un restaurateur? Est-ce que cette appréciation esthétique peut être fonction du degré ou de la qualité de lintervention du restaurateur? Cette question est évidemment indissociable d'une approche éthique : jusqu'où le 
restaurateur peut-il aller dans la recomposition? Doit-il laisser sa marque pour qu'on ne confonde pas la "vraie " nature et la nature restaurée ? Ces questions seront abordées dans le chapitre 4 . Pour ce qui est de leur dimension esthétique, disons simplement que ce que l'histoire nous a accoutumés à apprécier dans le paysage n'est jamais référé à une quelconque vérité écologique, même si toute élaboration paysagère est évidemment soumise à des contraintes écologiques, et si son degré d'achèvement est incontestablement corrélé à sa capacité d'intégrer ces contraintes dans la configuration sociale qui lui donne sens (comme en témoignent les exemples canoniques du bocage ou de l'habitat groupé méditerranéen).

\section{Un exemple de mise en spectacle : le Marais d'Orx}

A plusieurs titres, le Marais d'Orx constitue un cas exemplaire pour étudier le jeu complexe de la restauration écologique et de la mise en spectacle. De l'aveu même de l'instigateur principal de l'entreprise, le World Wildlife Fund, les premières opérations de restauration (si on laisse de côté le cas très particulier des réintroductions d'espèces), ont eu une dimension proprement esthétique. Il s'agissait, principalement pour les mines et les carrières, formes particulièrement violentes d'extraction des ressources naturelles, d'effacer les marques les plus stigmatisantes de l'action humaine : "Les carrières à ciel ouvert, les mines ou les sablières en sont l'illustration la plus connue et elles ont fourni les premiers exemples d'opérations de réparation. L'amélioration de l'esthétique de ces paysages dégradés est à l'origine des premiers travaux (rebouchage des trous) entrepris par les professionnels sous la pression de l'opinion puis des pouvoirs publics. Ces réparations, dans un premier temps purement mécaniques, se sont progressivement transformées en techniques de restauration voire de renumérotation " (WWF 1994, p. 4).

Qu'en est-il du domaine d'Orx, considéré à juste titre comme une réussite exemplaire de la restauration? L'opération a été menée de bout en bout par le WWF : son directeur scientifique, JeanFrançois Terrasse, avait lui-même découvert fortuitement le site au cours d'une promenade. "C'était en décembre 1984, en ballade dans les landes de Gascogne avec un ami ornithologue. J'ai aperçu une immense pièce d'eau qui s'étirait quasiment jusqu'à l'horizon. Seule, une route traversait cette étendue au ras de l'eau. J'ai tout de suite voulu savoir de quoi il s'agissait car il n'y avait pas de marais indiqué à cet endroit sur la carte. Très rapidement, j'ai appris que cette cuvette derrière les dunes littorales était jusqu'à l'automne précédent cultivée en maìs. Cet espace est intéressant car il est situé sur la principale ligne de migration d'Europe occidentale. C'est un refuge idéal pour de nombreux oiseaux migrateurs qui ne peuvent franchir les Pyrénées quand les conditions météorologiques sont défavorables" (PANDA 1993). Si le point de départ de l'opération réside dans une forte préoccupation ornithologique, appuyée dès ses premiers moments sur des savoirs et non sur une volonté d'aménagement, on ne peut pas dire pour autant qu'elle émane de la communauté scientifique. La restauration du Marais d'Orx apparaît plutôt comme la combinaison d'une passion ornithologique et de la capacité de coordination d'une grande organisation privée de protection de la nature.

La description rapide du montage de l'opération Marais d'Orx suffit à indiquer que la préoccupation de l'aménagement à des fins pédagogiques et "récréatives". Un dépliant publicitaire présente les choses dans le langage de l'équilibre irénique :

"Nourriture abondante et variée, grande tranquillité, le site accueille toute l'année d'innombrables hôtes. Chacun y trouve son compte :

- le balbuzard prélève quelques carpes, les spatules filtrent l'eau boueuse, la guifette picore de menus insectes tout en survolant la surface des flots, tandis que des milliers de canards barbotent de concert.

- certains oiseaux, à l'instar des grands goélands, s'y attardent quelques jours pour reprendre des forces ou en attendant l'accalmie d'une tempête atlantique. D'autres choisissent d'y rester, ils nichent dans les joncs des rivages ou se juchent sur un radeau de longues herbes ainsi que le font les grèbes.

Le marais bénéficie de conditions climatiques clémentes, si bien qu'en hiver il ne gèle jamais. C'est un refuge idéal pour les grues cendrées, les oies sauvages et les innombrables sarcelles, un site exceptionnel où se côtoient en toute quiétude l'homme et l'animal ".

Il ne faut pas surinterpréter ce texte, destiné à attirer un large public. Disons simplement que l'univers décrit est à la fois abondant ("innombrables " utilisé deux fois, "milliers de canards "). La prédation y est évoquée par petites touches mais le caractère modeste du prélèvement y est très nettement suggéré ("quelques carpes", "picore de menus insectes") : il s'agit en quelque sorte d'une alimentation de Weight Watchers. Une atmosphère de profusion et de paix enveloppe toute la scène : le Marais d'Orx apparait comme une hôtellerie bienfaisante où les oiseaux viennent reprendre des forces, et ils font bon ménage avec les hommes, dans la paix d'interactions contrôlées. La restauration écologique produit aussi une forme de pacification.

L'avant-projet sommaire d'aménagement du Marais d'Orx, da à l'intervention conjointe d'un ingénieur écologue et d'un architecte paysagiste (WWF 1993), a explicitement pour objectif un double aménagement écologique et paysager, les deux aspects étant complémentaires mais non confondus. Il s'agit de " recréer une zone humide d'importance européenne et de permettre le développement d'une activité culturelle éducative et touristique axée sur la découverte du patrimoine naturel, historique et du paysage " (p. 1). C'est que le site réunit deux caractéristiques rarement associées: le caractère de réserve et l'accessibilité. Le domaine d'Orx est en effet " proche de grandes voies de communication et de centres urbains importants, français et espagnols, ceci dans une région à très forte vocation touristique " (p. 3). Ceci impose, au-delà des dispositifs techniques qui assurent la maitrise à peu près complète des niveaux d'eau (par le recours en particulier à des stations de pompage), 
ce que les auteurs de l'avant-projet nomment sans ambiguité " une mise en scène" (p. 7).

Une telle mise en scène porte d'abord sur un certain nombre d'interventions concernant la nature, visant à la fois à la maitrise des eaux par pompage (exemple, fiche de projet 12 : "mise en place de groupes immergés de $2000 \mathrm{~m}^{3}$ ") et à la stimulation d'un certain nombre d'espèces privilégiées (exemple, fiche de projet 10 : "garantir des conditions de sécurité optimales pour accroître de manière spectaculaire les effectifs d'espèces grégaires"). Les dispositifs divers mis en place, qu'ils soient d'ordre technique ou paysager, ont pour but de contrôler les phénomènes et d'en augmenter, par des choix appropriés, la spectacularité. Mais il existe aussi des interventions plus spécifiquement paysagères, comme la mise en place de circuits de visite destinés à canaliser les visiteurs et en quelque sorte à contraindre leur regard, ou rapprochement de la faune et du public (exemple, fiche de projet 15: "garantir des conditions de stationnement et de reposoir suffisantes pour accroitre les stationnements et les effectifs d'espèces à faible distance des berges " ou encore fïche de projet 3 : "garantir des conditions de sécurité suffisantes pour accroître de manière spectaculaire les rassemblements et les effectifis de certaines espèces grégaires, permettre la vision pour le public non averti à des distances relativement réduites".

La nature est ici présentée dans l'immédiateté d'une relation et avec le caractère spectaculaire d'une haute productivité spontanée, même si celleci est techniquement et sélectivement orientée. L'insertion du site dans un parcours touristique suppose des gratifications qui s'apparentent à celles que l'on retire de la fréquentation de parcs de loisir, bien qu'elles portent sur des objets particuliers (savants et pédagogiques, mais toujours colorés et chatoyants). Cette situation d'offre de spectacle interdit notamment que l'on propose au spectateur occasionnel la norme du comportement ascétique, fait de longs parcours et de longues patiences, mais aussi d'apprentissages livresques, qui s'est peu à peu constituée comme modèle de la contemplation éclairée de la nature à travers l'émergence de la figure de l'observateur soucieux de protection. La nature doit s'offrir sans délai et sans contrainte excessive.

Le loisir moderne est caractérisé par une triple relation à la nature :

- mise à distance (neutralisation des dangers, préventions contre les excès, les débordements de la nature, mise en oeuvre d'un système d'assurances contre les risques qu'elle enveloppe)

- mise en spectacle (la perception de la nature est guidée par des canons esthétiques, comme en témoigne l'exemple de la forêt de Fontainebleau, et les premiers aménagements apparaissent comme des mises application de ces canons)

- mise en contact (le loisir est l'occasion d'affiner ses sensations, de développer une relation sensible au monde qui est dans son principe incomparable avec d'autres formes de rapport à la nature, notamment utilitaires)

On peut dire que les formes contemporaines de mise en spectacle de la nature liées à l'observation savante d'espèces, d'espaces ou de processus biologiques constituent un compromis entre la récréation hédoniste qui est au principe du loisir moderne (la nature s'offrant simultanément dans sa proximité et dans sa primitivité) et l'apprentissage ascétique caractéristique du développement de passions savantes pour la faune et la flore (celles-ci supposant des formes d'initiation, de longues heures d'attente ou d'approche, et le recours à des instruments : la nature ne s'y offre qu'après de multiples détours). Le cas du marais d'Orx a ceci de particulièrement intéressant qu'il vise deux publics : le grand public qui attend que la nature soit à la fois primitive et proche, qu'elle le dépayse dans un cadre familier (c'est à cette attente que répondent les divers aménagements qui facilitent ce que j’ai appelé la " mise en contact"); le public des connaisseurs (principalement ornithologues), qui vient y chercher une diversité et une densité d'oiseaux exceptionnelles. Une des questions est précisément de savoir à quelles conditions ces deux relations à la nature sont compatibles. C'est assurément la diffusion du savoir écologique qui permet la transformation tendancielle de la nature récréative en nature comme objet de connaissance ludique : mais la mise en spectacle qui en résulte est probablement porteuse de nouvelles contradictions.

\section{Savoir écologique ou art du faux ?}

Une des premières questions d'ordre éthique est évidemment celle qui touche à la légitimité du remplacement d'éléments de nature disparus ou très fortement dégradés. En effet, tous les objets ne sont pas remplaçables sur les mêmes bases. " $\mathrm{Si}$ je vous prête ma montre digitale ou ma Mitsubishi et que vous perdiez ou que vous endommagiez l'une ou l'autre, vous pouvez certainement les remplacer par une montre ou une voiture de qualité identique. A un autre extrême, vous ne pouvez certainement pas remplacer ma famille si vous portez la responsabilité de sa perte, parce que je vois ma famille, et la relation que j'entretiens avec elle, comme unique ", remarque le philosophe Alastair Gunn (GUNN 1991 p. 294). On peut faire cette remarque à propos de l'exemple de Gunn : si en toute rigueur les deux Mitsubishi ne sont pas identiques, leur caractère substituable tient au fait qu'elles sont fabriquées en série (et que le véhicule perdu fait actuellement l'objet d'une production). Qu'en est-il du caractère substituable de la valeur sentimentale d'un objet, qu'il soit unique ou de série ? Cette forme d'investissement sur l'objet ne constitue pas à proprement parler une de ses propriétés : on doit donc la laisser en dehors de l'analyse.

Peut-on remplacer un élément de nature qui a été perdu à l'exemple de la Mitsubishi selon Gunn ? Quelles sont les limites de l'analogie entre des biens de consommation (quelle que soit par ailleurs leur valeur monétaire ou leur statut social) et des biens naturels ? La distinction entre la famille et la voiture est fondée sur une seule propriété : l'unicité de la première. Il faut introduire dans la discussion la nature des objets et non pas seulement leur caractère unique ou reproductible. Les êtres humains ne sont pas seulement des pièces uniques: ils ont aussi pour caractéristique le fait de ne pas être assimilables à un produit (même ceux dont la naissance est le produit, au moins partiel, des effets du génie 
génétique), puisqu'ils sont des êtres moraux. Ils sont aussi, à la différence des objets auxquels ils sont comparés pour les besoins de la démonstration, des êtres naturels.

Pour Gunn, les espèces et la plupart des zones naturelles se situent "quelque part entre ces extrêmes ", c'est-à-dire entre la Mitsubishi et sa femme, ce qui suppose une sorte de gradient de substituabilité des êtres et des objets. La nature est-elle reproductible par un tiers ? Peut-on mimer la reproduction des écosystèmes?

Robert Eliot a posé les bases de la discussion éthique sur la restauration dans un article paru il y a quinze ans: Faking Nature reste la référence fondamentale dans ce domaine (ELLIOT 1995). Elliot défend l'argument selon lequel il existe des objections décisives à la problématique de la restauration de la nature, dans la mesure où le processus perturbation/restauration, qui postule un certain type de réversibilité, ne permet pas de retrouver la valeur naturelle ou la naturalité de la nature existant antérieurement à la modification d'un espace. Un tel point de vue suppose qu'on se donne un critère pour définir la naturalité de la nature: celle-ci ne suppose pas de degrés (nous sommes dans une logique de tout ou rien : ou bien, un espace est naturel, ou bien il a irréversiblement perdu cet attribut), puisque la seule définition opératoire de la nature est: ce qui n'a pas été modifié par l'homme. La séduction qu'exercent les espaces qu'on regroupe sous la catégorie de wilderness (NASH 1973), comme la valeur esthétique qu'ils recèlent et la possibilité de ressourcement moral qu'ils offrent est indiscutablement l'effet direct de cette nonanthropisation: des espaces jardinés, même lorsqu'ils présentent au moyen d'artifices des compositions dans lesquelles l'activité humaine a été complètement effacée, comme c'est le cas du parc à l'anglaise (WILLIAMS 1973) ne sont pas en mesure de produire ces effets. "Nous accordons de la valeur à la forêt et au fleuve en partie parce qu'ils sont représentatifs du monde qui échappe à notre domination, parce que leur existence ne dépend pas de nous" (ELLIOT p. 82). Les sentiments qu'inspire la nature sont d'un autre ordre que ceux que peut inspirer la vision de réalisations humaines de grande échelle (comme un barrage ou une ville).

Deux objections viennent immédiatement à l'esprit: la première, qui est aussi la plus générale, porte sur l'association entre naturalité et valeur éthique: la naturalité n'implique pas nécessairement de valeur. Elliot prend lui-même en compte cette objection en faisant remarquer que la maladie ou l'éruption volcanique, qui sont des phénomènes naturels, et qui altèrent néanmoins ce que nous valorisons dans la nature, ne contiennent pas ce type de valeur. Mais il ne va pas jusqu'au bout de son objection puisque cette concession ne le conduit pas à la remise en cause d'une définition unique de la naturalité. Or, il y a au moins ici deux formes de nature : celle qui se donne sous l'aspect d'un ordre naturel dont l'homme sait qu'il n'a pas contribué à sa constitution; celle qui apparaît comme désordre naturel ou comme pathologie. Il faut donc ajouter au caractère non-modifié de la nature envisagée par Elliot un second critère de la valorisation : celui de l'ordre. On en vient à la deuxième objection : l'affectation d'une valeur à des milieux non modifiés par l'homme doit être référée à deux types de conditionnements historiques. Les premiers portent sur la forme de nature valorisée. On sait que la valeur accordée à des paysages a beaucoup varié au cours de l'histoire et que l'appréciation de la notion de wilderness en tant que telle (c'est-à-dire en tant qu'elle exprime la manifestation d'une extériorité de la nature par rapport aux actions humaines) est à la fois récente dans l'histoire et qu'elle peut être localisée dans des conjonctures culturelles particulières (elle constitue un élément du sentiment moderne de la nature et, elle apparait dans sa formulation la plus précise comme une expression de l'histoire de la construction sociale de l'espace américain). Les seconds conditionnements portent sur la nature elle-même: sommes-nous sûrs que la valeur s'attache automatiquement à ce qui ne dépend pas de nous? La philosophie morale ne prend-elle pas trop rapidement à son compte le principe d'une extériorité de l'homme par rapport à la nature ? $\mathrm{Ne}$ valorisons-nous pas des espaces dotés de signification (et donc susceptibles d'investissements éthiques et esthétiques) parce qu'ils sont déjà partiellement anthropisés ou anthropisables? Un tel positionnement éthique ne correspond-il pas à un état antérieur de la science écologique? La théorie de la valeur de la naturalité défendue par Elliot exclut par principe ces questions.

Ce qui est perdu dans la réplication de la nature qu'autorise la restauration, la plus scrupuleuse soit-elle, c'est bien, selon Elliot, la dimension de la genèse, de l'histoire propre d'un site naturel. La naturalité d'un espace est perçue comme l'expression d'une forme de continuité historique, même si elle apparaît circonscrite dans les limites d'un dispositif de protection (comme dans un parc naturel) ou si elle est prise dans une politique de la nature : la continuité des chaînes causales garantit la naturalité. C'est ce qui explique que la réparation des dommages faits à la nature ne soit pas du même ordre que la prévention des dommages: seule la seconde garantit l'authenticité d'une politique aussi bien que l'authenticité de ses résultats. Protéger l'existant est justifié au nom de l'irréversibilité du processus de "dénaturation".

Les choses sont compliquées par le fait que la valeur paysagère d'un ensemble naturel n'est jamais ce qui constitue sa valeur selon les critères de l'éthique environnementale. Pourtant, valeur esthétique et valeur environnementale sont à l'état pratique difficiles à dissocier : les hauts lieux environnementaux sont aussi en règle générale de hauts lieux paysagers. Pour les spécialistes de l'éthique, il importe de rendre possible cette dissociation entre le paysage et la nature : pour Val Routley, la valorisation de l'état de wilderness ne peut jamais être réduite à une appétence pour la qualité paysagère. Ce qui est valorisé, c'est surtout la compréhension des mécanismes écologiques dont la perception paysagère constitue la traduction (ELLIOT 1995 p. 87). L'intellection l'emporte ici sur l'émotion. Le développement des savoirs écologiques apparaît ici comme une instance de production de nouvelles valeurs : les grilles de lecture de la nature que les biologistes mettent à disposition du public constituent de nouveaux cantons de la valeur: sont développés des critères inédits de jugement et d'évaluation des espaces perçus qui contribuent à réorienter le 
regard sur des espaces anciennement valorisés et surtout à conférer de la valeur à des espaces anciennement stigmatisés ou tenus pour négligeables (c'est le cas déjà évoqué des zones humides). A ce titre, l'écologie n'est passeulementsoumise à une interrogation éthique en tant qu'elle propose des définitions et des styles de traitement de la nature inédits : elle est aussi productrice de valeurs, qui ont nom complexité, diversité, intégration, par exemple. Leur pleine reconnaissance suppose une véritable conversion du regard que nous portons sur la nature.

Si l'on s'en tient au principe de l'irréversibilité stricte de la naturalité, on ne peut pas plus accepter l'idée d'une restauration de la nature que celle d'une restauration de la famille qu'on a perdue: on ne peut pas reproduire, même partiellement, les êtres chers. En conséquence, le génie écologique, qui n'est pas en mesure de reproduire du non reproductible, est une activité du même ordre que toute forme d'aménagement de l'espace : il obéit simplement à d'autres règles de procédure et à d'autres critères d'évaluation. Ceci n'implique pas qu'il soit par définition éthiquement condamnable: si les espaces restaurés ne sont pas donnés pour des morceaux de nature, mais que leur caractère d'artefact est explicité, ils peuvent avoir des fonctions positives (pédagogiques, récréatives et même écologiques). Ces réaménagements de morceaux de nature perdue pourraient être pensés par analogie avec l'encadrement ou l'agrandissement de portraits d'êtres qu'on a perdus, et qui contribuent à entretenir leur souvenir, et donc leur présence sur un autre mode. Ce qui peut être considéré comme une contrefaçon, c'est le fait de dire de ces constructions qu'il s'agit de nature.

Une position radicale dans ce domaine peut consister à ne pas accepter non plus les restaurations qui sont présentées comme telles (et qui ne constituent pas de ce fait des tromperies sur la marchandise ou des contrefaçons, puisqu'on les donne pour ce qu'elles sont), dans la mesure où elles finissent toujours par être confondues avec la nature pleinement naturelle. Je pense qu'on pourrait ici s'aider de la distinction, dans le domaine de l'œuvre d'art, entre le faux (qui constitue une tromperie délibérée) et la copie (qui se donne pour ce qu'elle est et qui peut avoir de nombreuses fonctions positives : diffusion de la connaissance de l'original, support pédagogique, etc...). Une position fondamentaliste conduira dans le domaine de la nature à déclarer illégitimes toutes les copies, dans la mesure où elles sont très peu faciles à distinguer du faux, puisqu'elles partagent les mêmes localisations, in situ . Une position nuancée conduira à reconnaitre les mérites de la copie pour toutes les vertus que nous avons évoquées.

Il apparaît à la lumière de cette analyse que l'écologie savante fait de plus en plus droit à l'historicité de ses propres objets, comme d'ailleurs d'autres secteurs de la biologie. Le terrain de la restauration est sans doute celui sur lequel la biologie et les sciences sociales sont amenées à faire des rencontres qui ne soient pas seulement protocolaires. La reconnaissance de la multiplicité des échelles d'observation aussi bien que l'affirmation du caractère "mortel" des configurations décrites qui installent ces savoirs dans une zone d'incertitude les rapproche du statut épistémologique que nous conférons généralement aux sciences sociales à la lecture des Essais sur la théorie de la science de Max Weber ou $\mathrm{du}$ Raisonnement sociologique de Jean-Claude Passeron. Il est de plus en plus couramment admis que les scientifiques peuvent proposer des réponses - ou à tous le moins des scénarios multiples à une question unique : il n'est pas rare qu'ils affirment être dans l'impossibilité de fournir une description univoque d'un processus. S'il existe très probablement un décalage entre l'image publique de l'activité scientifique (et partant un système d'attentes sociales) et ce que ces sciences fabriquent actuellement, il n'en reste pas moins que les débats concernant les enjeux environnementaux et leur gestion par la puissance publique contribuent à transformer progressivement la définition sociale de ce qu'on est en droit d'attendre de la production scientifique. Les échanges en cours sur le principe de précaution sont un bon exemple de ce déplacement. Les aspects immédiatement éthiques ou politiques de la discussion ne doivent pas dissimuler ses enjeux proprement épistémologiques: la mise en avant de l'incertitude n'est pas tant la reconnaissanee d'une forme d'impuissance du savoir rationnel qu'une redéfinition de ses critères de productivité. ...

Jean-Louis FABIANI EHESS 


\section{REFÉRENCES BIBLIOGRAPHIQUES}

- ARONSON J., 1997, "Les concepts et approches écologiques associés aux programmes de restauration et de réhabilitation ", Actes du séminaire de lancement du programme de recherche "Recréer la nature ", Paris, MNHN, p.p. 11-14.

- BARNAUD G., 1994, "A l'interface de la pratique et de la théorie : l'écologie de la restauration ", Natures, sciences, sociétés, vol. 3, p.p. 36-50.

- BERQUE A., 1995, Les raisons du paysage, Paris, Hazan.

- BLONDEL J., 1995, "Du théorique au concret : la biologie de la conservation ", Natures, sciences, sociétés, vol. 3, p.p. 10-18.

- BOWLES M.L. et WHELAN C.J., 1996, Restoration of Endangered Species. Conceptual Issues, Planning and Implementation, Cambridge, Cambridge University Press.

- CAIRNS J., 1991, "The status of the theoretical and applied science of restoration ecology ", The Environmental Professional, vol. 13, p.p. 186-194.

- ELLIOT R. (Éd), 1995, Environmental Ethics, Oxford, Oxford University Press.

- FABIANI J.L., 1985, "Science des écosystèmes et protection de la nature ", Cadoret A. (Dir), Protection de la nature. Histoire et idéologie, Paris, L'Harmattan, p.p. 75-107.

- FABIANI J.L., 1995, "Les recréateurs de la nature. Enjeu et justification d'une pratique paradoxale ", Natures, sciences, sociétés, hors-série, $n^{\circ} 3,1995$, p.p. 84-91.

- FABIANI J.L, 1996, "Principe de précaution et protection de la nature ", Le principe de précaution dans la conduite des affaires humaines, sous la direction d'Olivier Godard, Paris, MSH, p.p. 297-310.

- FABIANI J.L, 1996, "Haut lieu ou non lieu ?" La Petite mer. Portraits de l'étang de Berre Conservatoire du Littoral, p.p. 6-12.

- FABIANI J.L., 1999, "L'écologie de la restauration considérée comme mise en spectacle du patrimoine naturel ", Les Carnets $d u$ paysage, ${ }^{\circ} 4$, automne-hiver, p.p. 80-94.

- FABIANI J.L., 2000, "Éthique et politiques de la techno-nature. A propos de la biologie de la conservation ", Revue européenne des sciences sociales, tome XXXVIII, n ${ }^{\circ} 118$, p.p. 15-28.

- GUNN A., 1991, "The Restoration of Species and Natural Environments ", Environmental Ethics, Winter, p.p. 291-310.

- HOBBS R.J. et NORTON D.A., 1996, "Towards a Conceptual framework for Restoration Ecology ", Restoration Ecology, 4, p.p. 93-110.

- JORDAN W.R.III., GILPIN M.E. et ABER J.D., 1987, Restoration Ecology. A Synthetic Aprroach to Ecological Research, Cambridge, Cambridge University Press.

- JORDAN W., PETERS R.L. et ALLEN E.B., 1988, "Ecological restoration as a strategy for conserving biological diversity ", Environmental Management, vol. 12, p.p. 68-69.

- PANDA, 1993, "Zones humides, espaces remarquables en sursis ", n 53.

- PELOSSE V. et MICOUD A., 1993, «Du domestique au sauvage : des catégories pertinentes de la biodiversité ", Études rurales, n 129-130, p.p. 9-14.

- THOMAS K., 1985, Dans le jardin de la nature, Paris, Gallimard.

- WILLIAMS R., 1973, The Country and the City, Londres, Chatto and Windus.

- WWF, 1993, "Restauration et aménagement des Marais d'Orx ", avant-projet sommaire (J.J. Blanchon et F. Charmoy). 